\title{
Renal Disease Associated with Antiretroviral Therapy in the Treatment of HIV
}

\author{
Ryan D. Cooper ${ }^{\mathrm{a}}$ Marcello Tonelli ${ }^{\mathrm{a}-\mathrm{c}}$
}

Departments of a Medicine and ${ }^{\mathrm{b}}$ Public Health Sciences, and ${ }^{\mathrm{c}}$ Division of Critical Care Medicine, University of Alberta, Edmonton, Alta., Canada

\section{Key Words}

Renal disease $\cdot$ Antiretroviral therapy $\cdot$ HIV infection

\begin{abstract}
The introduction of potent combination antiretroviral therapy (ART) in the treatment of HIV infection has permitted reliable control of disease progression and has markedly improved survival among people with HIV. As a result, health care providers and patients have shifted clinical priorities; whereas once delaying opportunistic illness was a primary focus, increasing emphasis is now placed on preventative health, management of comorbid chronic disease and avoiding long-term toxicities of ART. Although renal disease is common in people with HIV, renal disease specifically due to ART remains relatively rare. Still, as the use of ART continues to increase, health care providers are likely to encounter this potentially serious complication with increasing frequency. Distinguishing ART-related nephrotoxicity from the myriad of other potential causes of renal disease in people with HIV is important in order to avoid unnecessary discontinuation of an appropriate ART regimen. This review focuses on the early recognition of renal disease associated with ART and suggests strategies for management and prevention.
\end{abstract}

Copyright ๑ 2011 S. Karger AG, Basel

\section{KARGER}

Fax +41613061234 E-Mail karger@karger.ch www.karger.com
(C) 2011 S. Karger AG, Basel

$1660-2110 / 11 / 1183-0262 \$ 38.00 / 0$

Accessible online at:

www.karger.com/nec

\section{Introduction: The Era of Potent Antiretroviral Therapy and Shift in HIV Clinical Priorities}

With the introduction of potent combination antiretroviral therapy (ART) has come the ability to durably and reliably control HIV disease progression. As a result, survival among people with HIV has improved dramatically and there is now hope for near-normal life expectancy [1-3].

The introduction of ART has also profoundly affected the course of HIV infection such that non-AIDS-related chronic conditions are replacing opportunistic illnesses as major causes of death and disease [1]. Renal disease, for example, is increasingly prevalent in people with HIV (up to $30 \%$ in some cohort studies) and now ranks as a major cause of death in this group [4-6].

The dramatic improvements in survival afforded by ART have also shifted the clinical priorities of health care providers and patients; whereas once delaying opportunistic illness was a primary focus, increasing emphasis is now placed on preventative health, management of comorbid chronic disease and avoidance of the long-term toxicities of ART [7].

Accumulating data from observational cohort studies suggest a number of potential long-term toxicities from ART, including lipoatrophy, neuropathy, dyslipidemia,

Dr. Marcello Tonelli

7-129 Clinical Science Building

8440112 St NW

Edmonton, AB T6B 2B7 (Canada)

Tel. +1 780407 8520, Fax +1 780407 7878, E-Mail mtonelli-admin@med.ualberta.ca 
glucose intolerance, osteopenia and cardiovascular disease [8]. This review focuses on ART-associated nephrotoxicity and suggests strategies for early clinical recognition and possible prevention.

Several excellent reviews on non-ART-related causes of renal injury in people with HIV have recently been published $[9,10]$. Reviews on antiretroviral dosage adjustment in chronic kidney disease (CKD) and end-stage renal disease (ESRD) are also available [11, 12]. Renal transplantation in people with HIV has also been reviewed [13].

\section{Renal Disease Is Common in HIV Patients but Is Usually due to Causes Other than ART}

Acute kidney injury (AKI) occurs frequently in people with HIV. The incidence of AKI in one contemporary cohort of ambulatory, community-based HIV patients was nearly 5.9 per 100 person-years, while in a separate study of hospitalized HIV patients, AKI complicated $6 \%$ of hospital stays $[14,15]$. Whether ambulatory or hospitalized, AKI in people with HIV is most commonly related to prerenal, ischemic or nephrotoxic insults in the context of advanced HIV disease and acute opportunistic illness. ART itself is only rarely implicated as a direct or contributing cause of AKI, and it probably occurs in less than $10 \%$ of cases.

CKD is also common in people with HIV, with prevalence rates up to 4 times that of the general population [16]. In HIV cohort studies, the prevalence of stage 3 or greater kidney disease is up to $10 \%$ [of note, since most studies relied on estimating equations such as the Modified Diet in Renal Disease Group or Cockcroft-Gault equations to estimate glomerular filtration rate (GFR), and since these equations can underestimate the actual GFR or creatinine clearance in patients with malnourishment or reduced muscle mass related to advanced HIV, it is possible that the true prevalence of CKD in these cohorts is underestimated]. Independent risk factors associated with CKD in people with HIV include older age, a history of previous opportunistic illness and the presence of relevant medical comorbidities such as diabetes and hypertension $[10,16]$.

In most urban North American surveys, African American race is the predominant risk factor for CKD, in part related to a racial predisposition to HIV-associated nephropathy (HIVAN). Although not strictly an AIDSdefining illness, HIVAN commonly (but not exclusively) occurs with advanced HIV. HIVAN typically improves with treatment of HIV, and thus HIVAN is now considered an independent indication for ART regardless of CD4 count [12].

The proportion of CKD in HIV patients specifically attributable to ART use appears to be relatively low [17]. Although correlations between CKD and exposure to specific antiretroviral agents have been observed in some cohort studies, the associations are substantially attenuated after adjustment for probable confounders such as older age and advanced HIV disease.

\section{Overall, ART Is Probably Beneficial for Kidney Function}

Initiation of effective ART is generally associated with improvements in renal function. This is especially apparent in HIV-infected persons with low CD4 counts and high HIV viral loads at the time of ART initiation and in those with preexisting kidney diseases such as HIVAN [18-20]. With the increasing availability of ART, the risk of ESRD in HIV patients has decreased by more than $50 \%$ in some populations [10]. Further, the clinical course of CKD has become indolent, and survival of HIV-infected persons with ESRD is prolonged. Parenthetically, this in turn has led to increasing prevalence of CKD in HIV populations despite declining incidence [10].

Improved renal function with administration of effective ART was directly illustrated in the landmark Strategies for Management of Antiretroviral Therapy trial [21]. This study aimed to better describe the balance between sustained control of HIV infection and cumulative exposure to potentially toxic antiretroviral agents. Thus, HIVinfected participants were randomized to receive either continuous ART or intermittent ART guided by current CD4 count (thus allowing ART 'holidays' and reduced total exposure to antiretroviral agents). The authors found significantly decreased all-cause mortality in those receiving continuous ART. Further, the incidence of renal disease and need for dialysis were significantly lower in patients receiving continuous ART compared to those on intermittent ART. The difference in renal outcomes was attributed to a direct role of unsuppressed HIV replication in causing progressive renal dysfunction $[10,20,21]$.

Thus, renal dysfunction per se should not be considered a contraindication to initiation of ART $[5,12]$. This point deserves special emphasis considering recent observations that HIV-infected persons with renal dysfunction may be less likely to receive ART than HIV patients without renal dysfunction $[4,22]$. 


\section{Despite the Salutary Effect of HIV Control on Renal Function, Some Individual Antiretroviral Agents Are Associated with Nephrotoxicity}

Notwithstanding the important benefits of ART on renal function, it must be recognized that specific antiretroviral agents can occasionally lead to reversible and irreversible renal injury. Indeed, isolated reports of nephrotoxicity exist for nearly all of the more than 20 currently available antiretroviral agents. However, a direct causal association remains unsubstantiated in many of these reports, while others attribute renal dysfunction to an antiretroviral agent only in the context of an idiopathic, systemic hypersensitivity reaction [23]. Many of the nucleoside reverse transcriptase inhibitors, especially older agents like didanosine, have been implicated as causes of type B lactic acidosis, but this acid-base imbalance is not, strictly speaking, renal toxicity. Only 3 antiretroviral agents have well-established associations with direct nephrotoxicity supported by numerous, consistent case reports and large cohort studies, namely indinavir, atazanavir and tenofovir disoproxil fumarate (TDF).

\section{Indinavir-Associated Nephrotoxicity: Common Side Effect but Obsolete Drug}

Indinavir is an antiretroviral from the protease inhibitor class and was among the first agents used as part of potent combination ART. Its efficacy in the suppression of HIV replication has been well documented in large, randomized, controlled trials. Indinavir was the most commonly prescribed protease inhibitor in 1996 but due to concerns of inconvenient dosing, meal restrictions and nephrolithiasis - combined with the availability of newer protease inhibitors with better tolerability profiles - it has now largely been replaced. Indeed, current HIV treatment guidelines specifically recommend against indinavir for initial therapy of HIV infection. Accordingly, indinavir is now only very rarely prescribed, if at all [12].

Indinavir is notorious for causing renal and urologic toxicity mediated by tubular crystallization [24-26]. Asymptomatic indinavir crystalluria is very common, possibly occurring in up to two thirds of treated individuals. However, the rate of clinically apparent indinavir nephrotoxicity is estimated at 6.7 per 100 person-years of indinavir use. Risk factors for overt disease include dehydration, increased indinavir serum concentration, low body weight and coadministration with acyclovir, trimethoprim-sulfamethoxazole and low-dose ritonavir.
Several different clinical presentations of indinavir nephrotoxicity are observed. Approximately $4 \%$ of treated patients will experience acute urolithiasis due to indinavir crystalluria, sometimes complicated by obstructive uropathy and acute renal failure. Because indinavir stones are radiolucent, secondary signs of obstruction on imaging studies in the context of flank pain and dysuria usually suggest the diagnosis.

Indinavir crystalluria can also present as a syndrome of flank pain and dysuria in the absence of frank urolithiasis. It is thought that crystal sludging results in tubular obstruction, with distension and/or bladder wall irritation then producing symptomatic disease.

Intermittent indinavir crystalluria can also lead to subacute or chronic tubulointerstitial nephritis. Although often reversible with prompt drug discontinuation, irreversible renal dysfunction with parenchymal fibrosis can also occur. Leukocyturia is commonly observed with indinavir-related interstitial nephritis. Indinavir crystals are characteristic on urine microscopy, with negative birefringence, a flat rectangular plate-like shape and wide size variation.

Indinavir (like all members of the protease inhibitor class) is extensively metabolized in the liver. However, unlike most other protease inhibitors, a significant amount of indinavir, up to $15 \%$, is also excreted unchanged by the kidney. (With the notable exception of atazanavir, all other HIV protease inhibitors have insignificant renal excretion). Indinavir is poorly soluble in urine at physiologic urine $\mathrm{pH}$.

The package insert and available clinical treatment guidelines recommend that individuals receiving indinavir drink at least 1.5 liters of water a day and that periodic urinalysis for pyuria and monitoring of serum creatinine concentration be performed. In some patients, indinavir has been resumed following uncomplicated nephrolithiasis, but switching instead to a better tolerated, more efficacious alternative antiretroviral agent seems a prudent management strategy.

\section{Atazanavir-Associated Nephrotoxicity: Widely Used Drug but Exceedingly Rare Side Effect}

Atazanavir is a newer antiretroviral agent of the protease inhibitor class. Several randomized trials have demonstrated its potent efficacy in controlling HIV infection. This, in combination with excellent tolerability and convenience of dosing, has led many clinical treatment guideline authorities to recommend atazanavir (especial- 
ly when used with low-dose ritonavir) as a preferred treatment for HIV [12].

Up to $8 \%$ of atazanavir is excreted unchanged via the kidney and, as for indinavir, the drug is poorly soluble in urine and especially likely to precipitate at alkaline $\mathrm{pH}$. However, in contrast to indinavir, clinically significant renal toxicity, crystalluria or nephrolithiasis were not observed in the initial clinical registration trials of atazanavir.

However, after widespread use in clinical settings, several reports of atazanavir nephrolithiasis were published [27]. Subsequently, a postmarketing review of the US Food and Drug Administration adverse event reporting system detected 12 additional confirmed cases of symptomatic nephrolithiasis due to atazanavir use [28]. A retrospective study of HIV patients from France found 11 cases of nephrolithiasis among 1,134 patients receiving atazanavir, suggesting a frequency of $0.97 \%$ [29]. Infrared spectrophotometry confirmed the presence of atazanavir-based urinary stones in each case. Specific risk factors were not identified [29]. In several of these cases, urologic intervention including percutaneous nephrostomy or ureteric stenting was required to reestablish normal renal function or relieve colic. Observed stones were radiolucent.

There is only one report of atazanavir crystalluria and associated interstitial nephritis in an HIV patient [30]; thus, this form of nephrotoxicity (commonly seen with indinavir) must be considered extremely rare with atazanavir.

Although the possibility of nephrolithiasis in HIV patients taking atazanavir-containing antiretroviral regimens must be recognized, specific preventive or monitoring strategies are not currently recommended. In most reported cases of nephrolithiasis, atazanavir was discontinued and a different ART regimen prescribed to replace it. However, in some cases, atazanavir was safely reintroduced with instruction to drink ample fluids of acidic $\mathrm{pH}$ (e.g. carbonated beverages); reported recurrence rates are low. Given the otherwise favorable tolerability profile and excellent efficacy of atazanavir, reinitiation may be considered in some cases, especially where other options are few or poorly tolerated.

\section{Tenofovir-Associated Nephrotoxicity: An Increasingly Recognized Rare Adverse Event of a Very Commonly Used Antiretroviral Agent}

TDF is an antiretroviral of the nucleotide reverse transcriptase inhibitor class. After several randomized controlled trials confirmed its potent efficacy as part of ART for control of HIV, TDF has become the most widely used antiretroviral worldwide.

TDF is generally considered safe and well tolerated because clinically important toxicities were rarely observed in phase III registration trials. However, since its increasing use in 'real-world' clinical settings and in less highly selected patient groups than typically seen in research trials, multiple case reports have linked TDF use with proximal renal tubulopathy, urinary phosphate wasting, decreased bone mineral density and impaired glomerular filtration [31, 32].

Several cohort studies have also associated modest rates of renal dysfunction with TDF use, with at least one study identifying a $1 \%$ yearly incidence of nephrotoxicity severe enough to warrant TDF discontinuation [33]. An industry-sponsored, postmarketing adverse events surveillance study found that over an estimated 400,000 person-years of TDF exposure, less than $0.2 \%$ of patients were judged to have experienced severe renal failure, although this outcome was not explicitly defined [34].

Review of reported cases of TDF-associated nephrotoxicity suggests that it most typically manifests as proximal tubular injury with associated reduction in glomerular filtration. Patients often develop glycosuria, tubular proteinuria, lowered serum phosphate and increased serum creatinine. Some patients develop frank Fanconi's syndrome and/or reduced bone mineral density. Rarely (perhaps in less than $2 \%$ of identified cases of toxicity) is dialysis required or symptomatic fracture experienced [34].

Risk factors for TDF-associated nephrotoxicity are not well established. Observational studies have suggested the importance of comorbid renal dysfunction, advanced age and coadministration of the antiretroviral didanosine. An association between TDF-related nephrotoxicity and protease inhibitor use has also been suggested but remains controversial $[35,36]$.

Figure 1 presents a schematic of a potential mechanism for TDF-related nephrotoxicity. TDF is renally excreted via a combination of glomerular filtration and active tubular secretion. In vitro studies have suggested that TDF is toxic to mitochondrial function in proximal convoluted tubule cells at high intracellular concentrations $[37,38]$. Presumably, disruption of mitochondrial function compromises tubular cell integrity, leading to tubule necrosis, Fanconi's syndrome and a decreased glomerular filtration rate. Intracellular accumulation of TDF may be influenced by tubular cell secretion by the multidrug resistance-associated protein transporter system, which in turn may be subject to competitive inhibition by pro- 
tease inhibitors such as ritonavir [39]. Didanosine coadministration with TDF may also lead to additive or even synergistic mitochondrial toxicity in the proximal convoluted tubule [39]. Renal biopsy in some HIV patients with clinical TDF-related nephrotoxicity has revealed tubular necrosis without glomerular or interstitial involvement.

The management of patients strongly suspected to have TDF-associated nephrotoxicity typically involves discontinuation of TDF and selection of an alternative ART regimen [32]. While several case reports found TDF-related nephrotoxicity to be reversible, a recently published case series from Australia found that $50 \%$ of patients achieved only partial recovery of renal function following discontinuation of TDF, although the authors could not identify correlates of incomplete renal recovery in these patients [40]. This emphasizes the importance of early recognition and accurate diagnosis of TDF-associated nephrotoxicity. The outcome of rechallenge in patients with confirmed TDF nephrotoxicity has only rarely been reported, and recommendations on this strategy cannot be made at this time.

An important strategy to prevent TDF nephrotoxicity is to ensure appropriate dose reduction in patients with preexisting renal dysfunction, especially as estimated GFR falls below $50 \mathrm{ml} / \mathrm{min}$ [32]. It also seems prudent to ensure that comorbid renal disease is fastidiously managed and that other nephrotoxic drugs are avoided in TDF recipients.

Recommendations for regular laboratory monitoring of patients on TDF vary. Most authorities suggest measurement of renal function (using either the CockcroftGault or Modified Diet in Renal Disease Group method for estimating GFR), serum phosphorus determination and urinalysis for protein and glucose semiannually [5]. Elevated fractional urinary excretion of phosphate is perhaps an earlier marker of proximal tubular dysfunction and might also be useful in monitoring for TDF toxicity.

While some authors suggest monitoring only those patients presumed at increased risk [i.e. those with preexisting renal dysfunction (estimated GFR $<90 \mathrm{ml} / \mathrm{min}$ ) who are either also taking other potentially nephrotoxic agents or who have relevant medical comorbidities such as diabetes or hypertension] [5], other authors recommend regular monitoring of all patients receiving TDF [12].

A recent randomized controlled trial of African patients receiving TDF-based ART found that routine laboratory monitoring of renal parameters did not improve rates of treatment success or reduce rates of treatment-

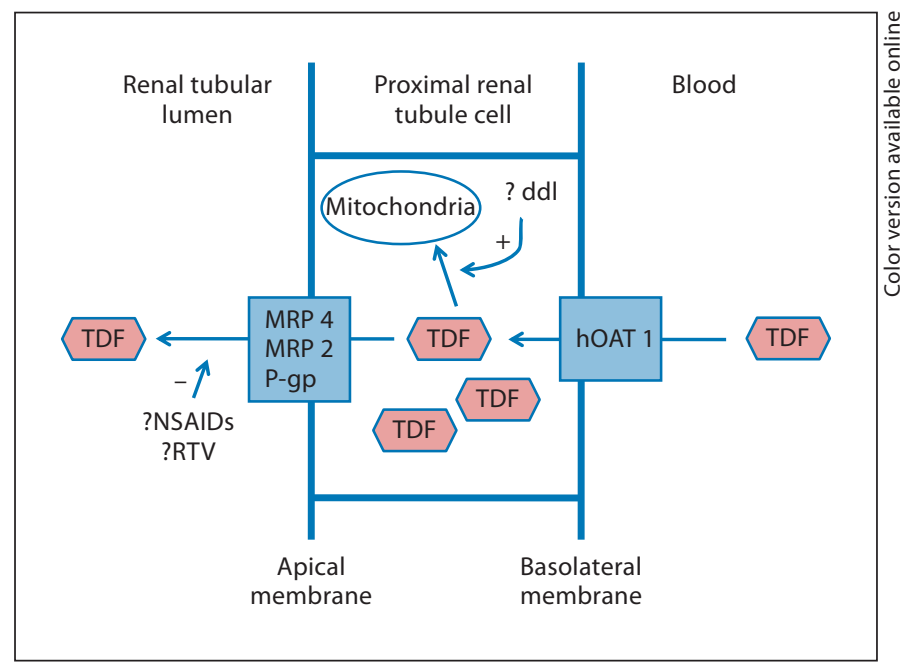

Fig. 1. Schematic diagram of the renal tubular secretion of TDF and potential intracellular accumulation with resultant mitochondrial toxicity in the presence of other pharmaceutical agents. See text for explanation and citations. hOAT $=$ Human organic anion transporter; $\mathrm{MRP}=$ multidrug resistance-associated protein; $\mathrm{P}$-gp = renal $\mathrm{P}$-glycoprotein; $\mathrm{ddI}=$ didanosine; NSAIDs = nonsteroidal antiinflammatory drugs; RTV = ritonavir.

related kidney disease [41]. However, this study excluded those with preexisting renal dysfunction, and the results may not be generalizable to patients with possible risk factors for TDF nephrotoxicity.

\section{It Is Not Enough to Simply Recognize ART-Related Nephrotoxicity - It Is Also Imperative to Distinguish it from Other Causes of Renal Injury}

Clearly, prompt recognition of antiretroviral nephrotoxicity is important if timely interventions to mitigate the extent of injury are to be implemented. In most cases of confirmed or definite antiretroviral nephrotoxicity, immediate drug discontinuation will be indicated.

However, it is also important for HIV care providers and consulting nephrologists to accurately diagnose ART nephrotoxicity and to distinguish it from other causes of renal injury in this population in order to avoid 'kneejerk' discontinuation of an otherwise effective antiretroviral regimen. Put differently, it is important to realize that ART nephrotoxicity accounts for the minority of cases of acute and chronic renal injury in HIV patients. Furthermore, although some HIV-related kidney diseases improve with successful ART (e.g. HIVAN), a beneficial response to ART has not been demonstrated for all HIV- 
related renal diseases (e.g. other forms of HIV-associated glomerulonephritis). Thus, for HIV patients on ART with persistent or progressive renal disease, renal biopsy is sometimes useful in establishing an accurate diagnosis.

Care providers must also appreciate the multiple trade-offs inherent in selecting an antiretroviral regimen - the balancing act between the need to maintain uninterrupted HIV suppression and the myriad of potential undesirable clinical effects and possible cumulative, nonrenal toxicities of alternative antiretroviral agents. In choosing alternative agents, it is also necessary to consider the possibility of acquired antiretroviral resistance with associated loss of antiretroviral efficacy further limiting possible treatment options. Any decision to discontinue or adjust ART in the face of apparent nephrotoxicity must be made in close collaboration between the HIV care provider and the consulting nephrologist.

\section{Conclusion}

The World Health Organization estimates that in 2009 almost 3 million people received ART. Since new guidelines recommend ART initiation earlier in the course of HIV infection, and HIV programs in resource-limited settings are 'scaling-up' their ART distribution, further dramatic increases in the number of global ART users are expected [42]. Thus, although long-term renal ART toxicity is relatively rare, health care providers are likely to encounter this potentially serious complication with increasing frequency. As individuals with HIV live longer and accumulate decades of experience with ART, it is possible that agents with even a modest propensity for nephrotoxicity may lead to more significant renal injury over time. Continued surveillance at the individual and population level may be helpful to rule out this possibility.

\section{References}

1 Lau B, Gange SJ, Moore RD: Risk of nonAIDS-related mortality may exceed risk of AIDS-related mortality among individuals enrolling into care with CD4+ counts greater than 200 cells $/ \mathrm{mm}^{3}$. J Acquir Immune Defic Syndr 2007;44:179-187.

-2 Palella FJ Jr, Baker RK, Moorman AC, Chmiel JS, Wood KC, Brooks JT, Holmberg SD; HIV Outpatient Study Investigators: Mortality in the highly active antiretroviral therapy era: changing causes of death and disease in the HIV outpatient study. J Acquir Immune Defic Syndr 2006;43:27-34.

-3 Bhaskaran K, Hamouda O, Sannes M, Boufassa F, Johnson AM, Lambert PC, Porter K; CASCADE Collaboration: Changes in the risk of death after HIV seroconversion compared with mortality in the general population. JAMA 2008;300:51-59.

4 Choi AI, Shlipak MG, Hunt PW, Martin JN, Deeks SG: HIV-infected persons continue to lose kidney function despite successful antiretroviral therapy. AIDS 2009;23:2143-2149.

$\checkmark 5$ Gupta SK, Eustace JA, Winston JA, Boydstun II, Ahuja TS, Rodriguez RA, Tashima KT, Roland M, Franceschini N, Palella FJ, Lennox JL, Klotman PE, Nachman SA, Hall SD, Szczech LA: Guidelines for the management of chronic kidney disease in HIV-infected patients: recommendations of the HIV Medicine Association of the Infectious Diseases Society of America. Clin Infect Dis 2005;40: 1559-1585.
6 Lucas GM, Mehta SH, Atta MG, Kirk GD, Galai N, Vlahov D, Moore RD: End-stage renal disease and chronic kidney disease in a cohort of African-American HIV-infected and at-risk HIV-seronegative participants followed between 1988 and 2004. AIDS 2007;21 2435-2443.

7 Phillips AN, Neaton J, Lundgren JD: The role of HIV in serious diseases other than AIDS. AIDS 2008;22:2409-2418.

$\checkmark 8$ Calmy A, Hirschel B, Cooper DA, Carr A: Clinical update: adverse effects of antiretroviral therapy. Lancet 2007;370:12-14.

$\checkmark 9$ Cohen SD, Chawla LS, Kimmel PL: Acute kidney injury in patients with human immunodeficiency virus infection. Curr Opin Crit Care 2008;14:647-653.

10 Winston J, Deray G, Hawkins T, Szczech L, Wyatt C, Young B: Kidney disease in patients with HIV infection and AIDS. Clin Infect Dis 2008;47:1449-1457.

11 Izzedine H, Launay-Vacher V, Deray G: Antiretroviral drugs and the kidney: dosage adjustment and renal tolerance. Curr Pharm Des 2004;10:4071-4079.

12 Panel on Antiretroviral Guidelines for Adults and Adolescents: Guidelines for the use of antiretroviral agents in HIV-1-infected adults and adolescents. 2009. http://www.aidsinfo. nih.gov/ContentFiles/AdultandAdolescentGL.pdf.

13 Gruber SA, Doshi MD, Cincotta E, Brown KL, Singh A, Morawski K, Alangaden G, Chandrasekar P, Losanoff JE, West MS, ElAmm JM: Preliminary experience with renal transplantation in HIV+ recipients: low acute rejection and infection rates. Transplantation 2008;86:269-274.
14 Wyatt CM, Arons RR, Klotman PE, Klotman ME: Acute renal failure in hospitalized patients with HIV: risk factors and impact on inhospital mortality. AIDS 2006;20:561-565.

15 Franceschini N, Napravnik S, Eron JJ Jr, Szczech LA, Finn WF: Incidence and etiology of acute renal failure among ambulatory HIV-infected patients. Kidney Int 2005;67: 1526-1531.

16 Overton ET, Nurutdinova D, Freeman J, Seyfried W, Mondy KE: Factors associated with renal dysfunction within an urban HIV-infected cohort in the era of highly active antiretroviral therapy. HIV Med 2009;10:343-350.

17 Fernando SK, Finkelstein FO, Moore BA, Weissman S: Prevalence of chronic kidney disease in an urban HIV infected population. Am J Med Sci 2008;335:89-94.

18 Reid A, Stöhr W, Walker AS, Williams IG, Kityo C, Hughes P, Kambugu A, Gilks CF, Mugyenyi P, Munderi P, Hakim J, Gibb DM; Development of Antiretroviral Therapy Trial: Severe renal dysfunction and risk factors associated with renal impairment in HIV-infected adults in Africa initiating antiretroviral therapy. Clin Infect Dis 2008;46:1271-1281.

19 Kalayjian RC, Franceschini N, Gupta SK, Szczech LA, Mupere E, Bosch RJ, Smurzynski M, Albert JM: Suppression of HIV-1 replication by antiretroviral therapy improves renal function in persons with low CD4 cell counts and chronic kidney disease. AIDS 2008;22: 481-487.

20 Mocroft A, Wyatt C, Szczech L, Neuhaus J, El-Sadr W, Tracy R, Kuller L, Shlipak M, Angus B, Klinker H, Ross M; INSIGHT SMART Study Group: Interruption of antiretroviral therapy is associated with increased plasma cystatin C. AIDS 2009;23:71-82. 
-21 Strategies for Management of Antiretroviral Therapy (SMART) Study Group, El-Sadr WM, Lundgren JD, Neaton JD, Gordin F, Abrams D, Arduino RC, Babiker A, Burman W, Clumeck N, Cohen CJ, Cohn D, Cooper D, Darbyshire J, Emery S, Fätkenheuer G, Gazzard B, Grund B, Hoy J, Klingman K, Losso M, Markowitz N, Neuhaus J, Phillips A, Rappoport C: CD4+ count-guided interruption of antiretroviral treatment. N Engl J Med 2006;355:2283-2296.

22 Choi AI, Rodriguez RA, Bacchetti P, Volberding PA, Havlir D, Bertenthal D, Bostrom A, O'Hare AM: Low rates of antiretroviral therapy among HIV-infected patients with chronic kidney disease. Clin Infect Dis 2007; 45:1633-1639.

23 Jao J, Wyatt CM: Antiretroviral medications: adverse effects on the kidney. Adv Chronic Kidney Dis 2010;17:72-82.

-24 Kopp JB, Miller KD, Mican JA, Feuerstein IM, Vaughan E, Baker C, Pannell LK, Falloon $\mathrm{J}$ : Crystalluria and urinary tract abnormalities associated with indinavir. Ann Intern Med 1997;127:119-125.

25 Kopp JB, Falloon J, Filie A, Abati A, King C, Hortin GL, Mican JM, Vaughan E, Miller KD: Indinavir-associated interstitial nephritis and urothelial inflammation: clinical and cytologic findings. Clin Infect Dis 2002;34: 1122-1128.

26 de Silva TI, Post FA, Griffin MD, Dockrell DH: HIV-1 infection and the kidney: an evolving challenge in HIV medicine. Mayo Clin Proc 2007;82:1103-1116.

-27 Anderson PL, Lichtenstein KA, Gerig NE, Kiser JJ, Bushman LR: Atazanavir-containing renal calculi in an HIV-infected patient. AIDS 2007;21:1060-1062.
-28 Chan-Tack KM, Truffa MM, Struble KA, Birnkrant DB: Atazanavir-associated nephrolithiasis: cases from the US Food and Drug Administration's Adverse Event Reporting System. AIDS 2007;21:1215-1218.

29 Couzigou C, Daudon M, Meynard JL, BorsaLebas F, Higueret D, Escaut L, Zucman D, Liotier JY, Quencez JL, Asselah K, May T, Neau D, Vittecoq D: Urolithiasis in HIV-positive patients treated with atazanavir. Clin Infect Dis 2007;45:e105-e108.

30 Brewster UC, Perazella MA: Acute interstitial nephritis associated with atazanavir, a new protease inhibitor. Am J Kidney Dis 2004; 44:e81-e84.

31 Woodward CL, Hall AM, Williams IG, Madge S, Copas A, Nair D, Edwards SG, Johnson MA, Connolly JO: Tenofovir-associated renal and bone toxicity. HIV Med 2009;10:482-487.

>32 Sax PE, Gallant JE, Klotman PE: Renal safety of tenofovir disoproxil fumarate. AIDS Read 2007;17:90-92, 99-104, C3.

33 Gallant JE, Parish MA, Keruly JC, Moore RD Changes in renal function associated with tenofovir disoproxil fumarate treatment, compared with nucleoside reverse-transcriptase inhibitor treatment. Clin Infect Dis 2005;40: 1194-1198.

34 Gupta SK: Tenofovir-associated Fanconi syndrome: review of the FDA adverse event reporting system. AIDS Patient Care STDS 2008;22:99-103.

-35 Gallant JE, Moore RD: Renal function with use of a tenofovir-containing initial antiretroviral regimen. AIDS 2009;23:1971-1975.

36 Goicoechea M, Liu S, Best B, Sun S, Jain S, Kemper C, Witt M, Diamond C, Haubrich R, Louie S; California Collaborative Treatment Group 578 Team: Greater tenofovir-associated renal function decline with protease inhibitor-based versus nonnucleoside reversetranscriptase inhibitor-based therapy. J Infect Dis 2008;197:102-108.
37 Vidal F, Domingo JC, Guallar J, Saumoy M, Cordobilla B, Sánchez de la Rosa R, Giralt M, Alvarez ML, López-Dupla M, Torres F, Villarroya F, Cihlar T, Domingo P: In vitro cytotoxicity and mitochondrial toxicity of tenofovir alone and in combination with other antiretrovirals in human renal proximal tubule cells. Antimicrob Agents Chemother 2006;50: 3824-3832.

38 Côté HC, Magil AB, Harris M, Scarth BJ, Gadawski I, Wang N, Yu E, Yip B, Zalunardo N, Werb R, Hogg R, Harrigan PR, Montaner JS: Exploring mitochondrial nephrotoxicity as a potential mechanism of kidney dysfunction among HIV-infected patients on highly active antiretroviral therapy. Antivir Ther 2006;11:79-86

>39 Zimmermann AE, Pizzoferrato T, Bedford J, Morris A, Hoffman R, Braden G: Tenofovirassociated acute and chronic kidney disease: a case of multiple drug interactions. Clin Infect Dis 2006;42:283-290.

40 Wever K, van Agtmael MA, Carr A: Incomplete reversibility of tenofovir-related renal toxicity in HIV-infected men. J Acquir Immune Defic Syndr 2010;55:78-81.

41 Mugyenyi P, Walker AS, Hakim J, et al: Routine versus clinically driven laboratory monitoring of HIV antiretroviral therapy in Africa (DART): a randomised non-inferiority trial. Lancet 2010;375:123-131.

42 Joint United Nations Programme on HIV/ AIDS (UNAIDS) and World Health Organization (WHO): AIDS Epidemic update. November 2009. http://www.who.int/hiv/pub/ epidemiology/epidemic/en/index.html (accessed 2010).

\section{Editorial Comment}

Meguid El Nahas, Sheffield

The review by Cooper and Tonelli is timely, as new guidelines recommend ART initiation earlier in the course of HIV infection with an expected global rise in the number of ART users. ART treatment relies to a large extent on protease inhibitors and drugs of the nucleotide reverse transcriptase inhibitor class.

The authors highlight the potential nephrotoxicity of these agents but also urge those treating HIV-positive individuals to discriminate between drug-related nephrotoxicity and other causes of HIV-associated kidney injury. They also stress that renal dysfunction should not be considered a contraindication to initiation of ART. How- ever, it is important to bear in mind that kidney function at ART initiation is an independent predictor of death in HIV-infected individuals, especially in those with a history of AIDS. There is little doubt that close monitoring of renal function is essential to minimize complications and improve outcomes in HIV-infected individuals. Kidney damage related to antiretroviral therapy is typically reversible with early recognition and timely discontinuation of the offending agent. Nephrologists should be familiar with the potential toxicity of these agents to avoid delays in diagnosis. 